\title{
Effects of Temperature and Separation on the Retained Coagulability of Human Blood Utilizing Thromboelastography
}

\author{
Michael A. Wiley, $\mathrm{MS}^{1}$ and Nathan J. Alves, $\mathrm{PhD}^{2}$ \\ ${ }^{1}$ Indiana University School of Medicine, ${ }^{2}$ Indiana University School of Medicine, \\ Department of Emergency Medicine
}

\section{Background and Hypothesis:}

Thromboelastography is torsional method of assessing coagulation efficiency (e.g. rate of clot formation and maximum clot strength) by measuring the viscosity changes of blood under low-shear stress. Limited research has been performed to date on how centrifugation and storage of whole blood affects the coagulability when recombined. We hypothesized that centrifuged blood that was stored at its component-specific ideal temperatures would produce a clot with the same clotting characteristics as whole blood.

\section{Experimental Design or Project Methods:}

Whole blood samples were collected from healthy volunteers into citrated tubes and were assigned to the following conditions: whole at $25^{\circ} \mathrm{C}$, whole at $4^{\circ} \mathrm{C}$, and centrifuged and stored as RBCs, platelets, and plasma (at $4^{\circ} \mathrm{C}, 25^{\circ} \mathrm{C}$, and $-20^{\circ} \mathrm{C}$, respectively) and then recombined. TEG tracings for each condition were obtained over the course of three weeks. We also explored how freezing and thawing whole blood samples and its components affected clotting.

\section{Results:}

When compared to room temperature and refrigerated whole blood, separated blood maintains its normal physiologic clotting dynamics and kinetics for a longer period of time. Whole blood at $25^{\circ} \mathrm{C}$ maintains maximum clot strength for approximately one week post draw, whereas freezing whole blood drastically reduces coagulability. Additionally, we discovered that there is no practical difference in the clotting parameters between the first blood draw and subsequent draws.

\section{Conclusion and Potential Impact:}

Our study demonstrates the viability of storing blood in its components and recombining them at a later date while minimally affecting its clotting ability, thereby potentially reducing wasted blood products and the need for multiple blood draws for coagulation studies. 